\title{
Detection of secondary eclipses of WASP-10b and Qatar-1b in the Ks band and the correlation between Ks-band temperature and stellar activity.
}

\author{
Patricia Cruz $^{1,2}$, David Barrado ${ }^{2}$, Jorge Lillo-Box ${ }^{3,2}$, Marcos Diaz ${ }^{1}$, \\ Mercedes López-Morales ${ }^{4}$, Jayne Birkby ${ }^{4,5}$ Jonathan J. Fortney $^{6}$ \\ and Simon Hodgkin ${ }^{7}$, \\ ${ }^{1}$ Instituto de Astronomia, Geofísica e Ciências Atmosféricas, Universidade de São Paulo \\ (IAG/USP), Brazil \\ email: patricia.cruz@usp.br \\ ${ }^{2}$ Departamento de Astrofísica, Centro de Astrobiología (CAB/INTA-CSIC), Spain \\ ${ }^{3}$ European Southern Observatory (ESO), Chile \\ ${ }^{4}$ Harvard-Smithsonian Center for Astrophysics, USA \\ ${ }^{5}$ NASA Sagan Fellow \\ ${ }^{6}$ Department of Astronomy and Astrophysics, University of California, USA \\ ${ }^{7}$ Institute of Astronomy, University of Cambridge, UK
}

\begin{abstract}
The Calar Alto Secondary Eclipse study was a program dedicated to observe secondary eclipses in the near-IR of two known close-orbiting exoplanets around K-dwarfs: WASP$10 \mathrm{~b}$ and Qatar-1b. Such observations reveal hints on the orbital configuration of the system and on the thermal emission of the exoplanet, which allows the study of the brightness temperature of its atmosphere. The observations were performed at the Calar Alto Observatory (Spain). We used the OMEGA2000 instrument (Ks band) at the 3.5m telescope. The data was acquired with the telescope strongly defocused. The differential light curve was corrected from systematic effects using the Principal Component Analysis (PCA) technique. The final light curve was fitted using an occultation model to find the eclipse depth and a possible phase shift by performing a MCMC analysis. The observations have revealed a secondary eclipse of WASP-10b with depth of $0.137 \%$, and a depth of $0.196 \%$ for Qatar- $1 \mathrm{~b}$. The observed phase offset from expected mideclipse was of -0.0028 for WASP-10b, and of -0.0079 for Qatar- $1 \mathrm{~b}$. These measured offsets led to a value for $|e \cos \omega|$ of 0.0044 for the WASP-10b system, leading to a derived eccentricity which was too small to be of any significance. For Qatar-1b, we have derived a $|e \cos \omega|$ of 0.0123 , however, this last result needs to be confirmed with more data. The estimated Ks-band brightness temperatures are of $1647 \mathrm{~K}$ and $1885 \mathrm{~K}$ for WASP-10b and Qatar-1b, respectively. We also found an empirical correlation between the $\log \left(R_{\mathrm{HK}}^{\prime}\right)$ activity index of planet hosts and the Ks-band brightness temperature of exoplanets, considering a small number of systems.
\end{abstract}

Keywords. Planetary systems, stars, photometry

\section{Introduction and data reduction}

The Calar Alto Secondary Eclipse study (the CASE study) was an observational program dedicated to observe in the near-infrared, from the ground, secondary eclipses of two known close-orbiting exoplanets: WASP-10b (Christian et al. 2009) and Qatar-1b (Alsubai et al. 2011). Such observations reveal hints on the orbital configuration of the system and on the thermal emission of the exoplanet.

The observations were performed in the Ks band with the OMEGA2000 instrument mounted on the $3.5 \mathrm{~m}$ telescope of the Calar Alto Observatory (CAHA), in Spain. The 
telescope was strongly defocused, resulting in a ring-shaped PSF. The data were gathered in staring mode, where we observed the target continuously without any dithering during several hours. Before and after the staring mode sequence, we also obtained in-focus images composed by five dither-point images each with the purpose of obtaining sky images for further subtraction.

The initial data reduction was performed using IRAF for the bad pixel removal, flatfielding, and sky subtraction. We constructed a normalized sky map based on the in-focus images, which was scaled to the observed median background of each image and then subtracted. A circular aperture photometry was done in IDL for the target and for all sufficiently bright stars in the field of view. Only the stars that did not present any strong variations or other odd behavior in their light curves were used as reference for the differential photometry.

\section{Secondary eclipse analysis}

\subsection{The CASE study: WASP-10b}

We observed the planet-host WASP-10 during approximately 5.4 continuous hours. After the reduction described above, we used the Principal Component Analysis (PCA) technique to identify parameters related to visible trends in the differential light curve, revealing significant correlations of the normalized flux with the star's y-position at the detector, defocused seeing, airmass, and background count level. We fit for these systematics simultaneously by performing a multiple linear regression, where we modeled the trends only on the out-of-eclipse portions of the light curve, applied the model to the in-eclipse portion, and removed it from the light curve. The complete description of the reduction procedure and analysis performed can be found in Cruz et al. (2015).

Firstly, we binned the light curve with 127 points per bin, and performed a fit to obtain the secondary eclipse depth and detect a possible phase offset by using the occultation model from Mandel \& Agol (2002), assuming no limb-darkening. In order fit for the expected phase of mid-eclipse $\phi_{c}$, the depth of the secondary eclipse $\Delta F$, and the out-ofeclipse baseline level $F_{\mathrm{bl}}$, we performed a Markov chain Monte Carlo (MCMC) method, generating four chains of $1 \times 10^{6}$ simulations each. After combining the results from all chains (total of $4 \times 10^{6}$ simulations), the best model obtained from the MCMC analysis was the one with $\Delta F$ of $0.137 \%_{-0.019 \%}^{+0.013 \%}, \Delta \phi$ of $-0.0028_{-0.0004}^{+0.0005}$, and $F_{\mathrm{bl}}=0.99984_{-0.00010}^{+0.00008}$ (fig. 1). We also fit for the systematics and the occultation model simultaneously. For that, we applied the MCMC analysis to the unbinned light curve with 3900 individual measurements. With the baseline level fixed at $F_{\mathrm{bl}}=1.0$, this analysis provided the following results: $\Delta F=0.139 \%_{-0.023 \%}^{+0.011 \%}$ and $\Delta \phi=-0.0019_{-0.0002}^{+0.0019}$.

\section{Derived orbital configuration}

The measured offset of $\Delta \phi=-0.0028$ means that the center of the secondary eclipse occured at phase $\phi_{e c l}=0.4972$ instead of at phase $\phi=0.5$, expected for a circular orbit. From the results, we obtain that $e \cos \omega \simeq-0.0044$. The negative sign indicates that $\omega$ is a value between 90 and 270 degrees. Considering the $\omega$ of $167.13^{\circ}$, published by Christian et al. (2009), its orbit would have an eccentricity of $e \simeq 0.0045$. This value is fully consistent with a circular orbit, although it is in disagreement with their published eccentricity of $e \simeq 0.059$. Instead, if we use the value given by Johnson et al. (2009) for $\omega$ of $153.3^{\circ}$, we get $e \simeq 0.0049$, also in favor of a null eccentricity and different from their result $(e \simeq$ 0.051). Nevertheless, these derived eccentricities are too small to be of any significance. 


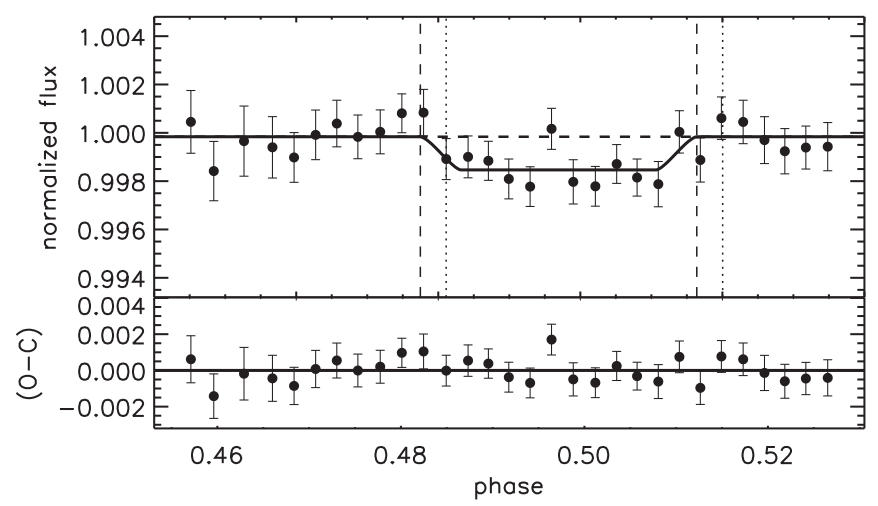

Figure 1. Binned light curve (127 points per bin) showing the secondary eclipse of WASP-10b in the Ks band. The best-fitting model (solid line) provided an eclipse depth of $\Delta F=0.137 \%$. The ingress and egress positions of the expected eclipse, considering a circular orbit, are represented by dotted lines, and the dashed lines show a phase shift of $\Delta \phi=-0.0028$. In the lower panel, the residuals are shown. (Cruz et al. 2015, fig. 3.)

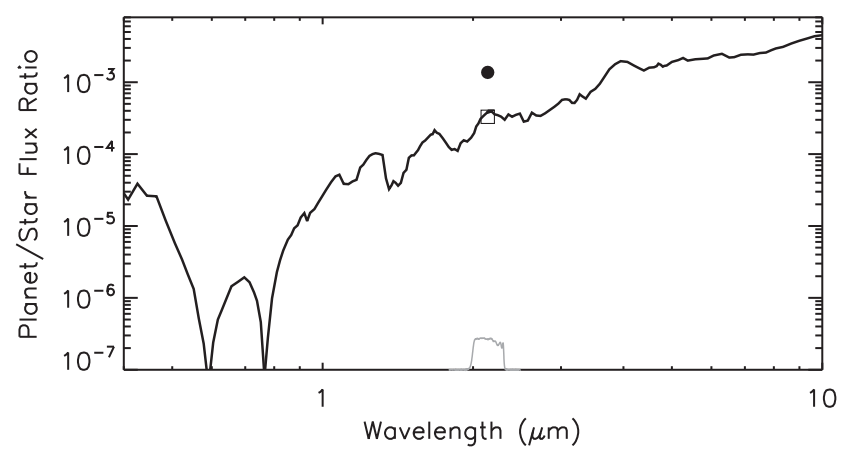

Figure 2. Model spectrum of thermal emission of WASP-10b computed without $\mathrm{TiO} / \mathrm{VO}$ and considering $f=2 / 3$. The measured planet-to-star flux ratio (at $2.14 \mu \mathrm{m}$ ) is represented by a filled circle (the error bars are inside the filled circle). The expected flux considering the presented model is represented by a square. The Ks-band transmission curve (gray line) is shown at the bottom of the panel at arbitrary scale. (Cruz et al. 2015, fig. 8.)

\section{Estimated thermal emission}

The MCMC analysis resulted in a planet-to-star flux ratio of $0.137 \%$, which is given by the eclipse depth. Assuming both components of the system, planet and star, emit as black-bodies, we derived a Ks-band brightness temperature for WASP-10b of $T_{\mathrm{Ks}} \simeq$ $1647_{-131}^{+97} \mathrm{~K}$ (Cruz et al. 2015). The maximum expected equilibrium temperature for the planet, assuming zero Bond albedo and instant reradiation $\left(A_{B}=0\right.$ and $f=2 / 3$, respectively) is $T_{\text {eq }} \simeq 1224 \mathrm{~K}$. This temperature is about $25 \%$ cooler than the observed $T_{\mathrm{Ks}}$.

From the comparision of the measured planet-to-star flux ratio to several atmospheric spectral models, we noticed that none of the models used was able to reproduce the high emission of WASP-10b in the Ks band. We used the models by Fortney et al. (2006, 2008 ), generated with different reradiation factors $(f)$ and computed without $\mathrm{TiO} / \mathrm{VO}$ (models without $\mathrm{TiO} / \mathrm{VO}$ tend to have higher K-band fluxes). The model which produces the highest emission possible in the observed band considers an instant reradiation over the dayside $(f=2 / 3)$ and it is shown in figure 2 . 


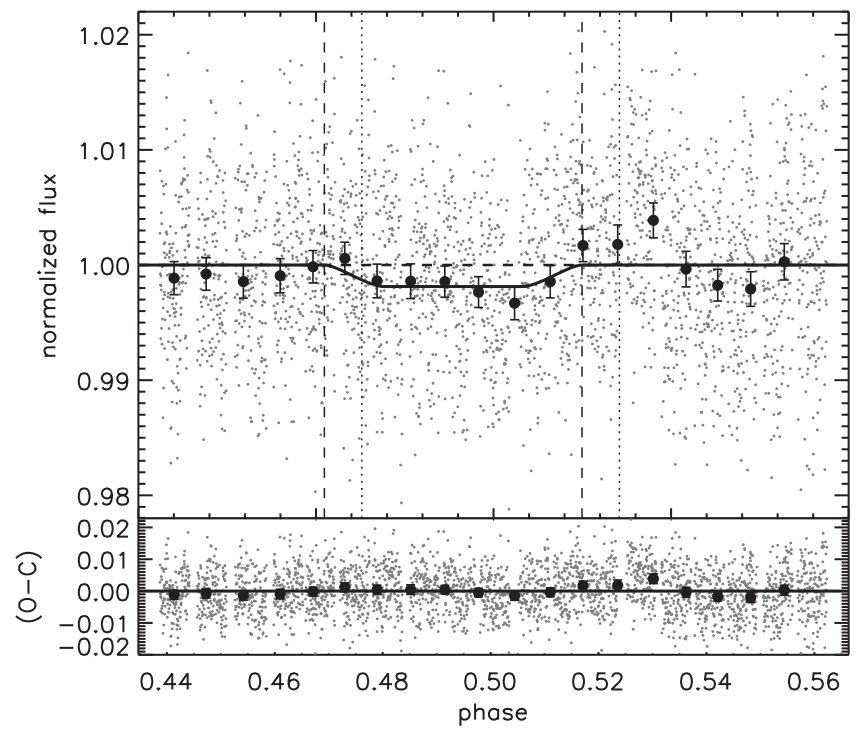

Figure 3. Detrended light curve of the secondary eclipse of Qatar-1b in the Ks band. The best-fitting model (solid line) resulted in $\Delta F=0.186 \%$, and $\Delta \phi=-0.0071$. The individual measurements are represented by small dots, and the filled circles represent the same data binned every 143 points, for better visualization. The expected ingress and egress positions, considering circular orbit, are shown as dotted vertical lines. The dashed lines represent the phase shift provided by the analysis. (Cruz et al. 2016, fig. 3.)

\subsection{The CASE study: Qatar-1b}

We observed the planet-host Qatar-1 during approximately 4.2 continuous hours. The data reduction followed the same procedure described earlier. The differential light curve generated was also corrected from systematic effects by using the PCA technique, presenting significant correlations of the normalized flux with star's xy-position, defocused seeing, and airmass. The details on the reduction and analysis are describde in Cruz et al. (2016).

The final decorrelated light curve was fitted using the occultation model from Mandel \& Agol (2002), assuming no limb-darkening, by performing a MCMC analysis. We then performed the MCMC analysis of the uncorrected light curve (with 2850 individual measurements), unifying the decorrelation function and the occultation model in a joint fit, with the objective of integrating the effect of the systematics into the uncertainty estimates. The best fitting model resulted in $\Delta F=0.186 \%_{-0.024 \%}^{+0.022 \%}$, and $\Delta \phi=-0.0071_{-0.0010}^{+0.0006}$, with $F_{\text {bl }}$ fixed at 1.0 (fig. 3).

Since the red noise present in these data is not negligible, we have used the "Prayer Bead" method (Moutou et al. 2004, Gillon et al. 2007) to assess the impact of red noise on the derived parameters and errors, which resulted in revised values for $\Delta F$ and $\Delta \phi$ of $0.196 \%_{-0.051 \%}^{+0.071 \%}$ and $-0.0079_{-0.0043}^{+0.0162}$, respectively. It is worth mentioning that the sample of red noise we have is limited, and its timescale is comparable with the eclipse duration, which could have compromised this final analysis and led to an overestimation of the derived uncertainties. 


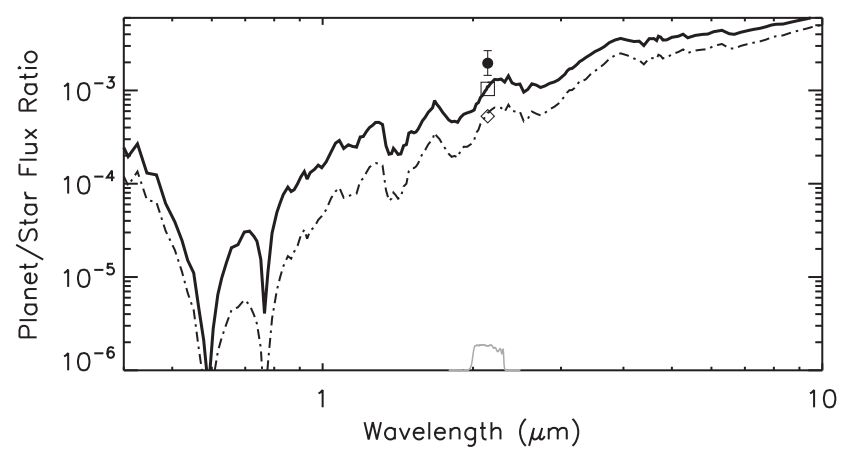

Figure 4. Model spectrum of thermal emission of Qatar-1b computed without $\mathrm{TiO} / \mathrm{VO}$ and $f=2 / 3$ (solid line). The measured planet-to-star flux ratio is represented by a filled circle. For comparison, a similar model with $f=1 / 2$ is presented (dot-dashed line). The Ks-band transmission curve (gray line) is also shown, at arbitrary scale. (Cruz et al. 2016, fig. 5.)

\section{Derived orbital configuration}

The measured offset in phase led to a derived $e \cos \omega$ of $-0.0123_{-0.0067}^{+0.0252}$. Due to the large uncertainty of $\Delta \phi$, the estimated $e \cos \omega$ can lead to several combinations of $e$ and $\omega$, making impossible to constrain $\omega$ for eccentricities lower than 0.02 (see Cruz et al. 2016 for more details). Additional data are needed to further investigate the orbital configuration of this exoplanet.

\section{Estimated thermal emission}

From the $\Delta F$ derived in the analysis, and assuming that Qatar-1b and its central star emit as black-bodies, we estimated a Ks-band brightness temperature of $T_{\mathrm{Ks}} \simeq 1885_{-168}^{+212}$ K. Considering an instant reradiation $(f=2 / 3)$ and a zero Bond albedo $\left(A_{B}=0\right)$, we would expect a maximum equilibrium temperature for this exoplanet of $T_{\text {eq }} \simeq 1768 \mathrm{~K}$ (Cruz et al. 2016). Then, the estimated $T_{\mathrm{Ks}}$ of Qatar-1b is in accordance with $T_{\mathrm{eq}}$, within the error estimates.

We then compared the measured planet-to-star flux ratio with several atmospheric spectral models by Fortney et al. (2006, 2008). As done previously, these models were computed with different reradiation factors, and without $\mathrm{TiO} / \mathrm{VO}$. Figure 4 shows the atmospheric model that better reproduces the observed emission of Qatar-1b, with an inefficient heat redistribution $(f=2 / 3)$.

\section{Discussion}

\subsection{Presence or absence of thermal inversions}

A thermal inversion could be caused due to the presence of an opacity source in the atmosphere of an exoplanet. For instance, some studies have suggested that $\mathrm{TiO}$ and VO present in a high layer of the atmosphere could lead to a hot stratosphere and to a temperature inversion. According to Fortney et al. (2006), atmospheric models with such an inversion would show a weak emission in the near-infrared (JHK bands), however, models without this thermal inversion would have stronger emissions. From our analyses presented earlier, the atmospheric models that most approximate the planet-to-star flux ratio measured for WASP-10b and Qatar-1b are models computed without TiO/VO. This suggests that both exoplanets whould have atmospheres without a thermal inversion layer. 
Table 1. Secondary eclipses (SE) in the Ks band from the literature.

\begin{tabular}{lccc}
\hline \hline Object & SE depth $(\%)$ & $T_{\mathrm{Ks}}(\mathrm{K})$ & Reference \\
\hline CoRoT-1b & $0.336 \pm 0.042$ & $2460_{-160}^{+80}$ & Rogers et al. 2009 \\
CoRoT-2b & $0.16 \pm 0.09$ & $1890_{-350}^{+260}$ & Alonso et al. 2010 \\
HAT-P-1b & $0.109 \pm 0.025$ & $2136_{-170}^{+150}$ & de Mooij et al. 2011 \\
Qatar-1b & $0.196_{-0.051}^{+0.071}$ & $1885_{-168}^{+212}$ & Cruz et al. 2016 \\
TrES-2b & $0.062_{-0.011}^{+0.013}$ & $1636_{-88}^{+79}$ & Croll et al. $2010 \mathrm{a}$ \\
TrES-3b & $0.133_{-0.016}^{+0.018}$ & $1731_{-60}^{+56}$ & Croll et al. $2010 \mathrm{~b}$ \\
WASP-3b & $0.181 \pm 0.020$ & $\sim 2435$ & Zhao et al. 2012 \\
WASP-4b & $0.185_{-0.013}^{+0.014}$ & $1995 \pm 40$ & Cáceres et al. 2011 \\
WASP-10b & $0.137_{-0.019}^{+0.013}$ & $1647_{-131}^{+97}$ & Cruz et al. 2015 \\
WASP-12b & $0.309_{-0.012}^{+0.013}$ & $2988_{-46}^{+45}$ & Croll et al. 2011 \\
WASP-19b & $0.287 \pm 0.020$ & $2310 \pm 60$ & Zhou et al. 2014 \\
\hline \hline
\end{tabular}

Fortney et al. (2008) and Burrows et al. (2008) proposed that highly irradiated exoplanets, with $F_{\text {inc }}>10^{9} \mathrm{erg} \mathrm{s}^{-1} \mathrm{~cm}^{2}$, would present a thermal inversion layer in their atmospheres, however, at low irradiation levels, the exoplanetary atmospheres would not show temperature inversions. Both objects studied, WASP-10b and Qatar-1b, fall in the category of low irradiated exoplanets, with $F_{\text {inc }} \sim 0.2 \times 10^{9}$ and $F_{\text {inc }} \sim 0.8 \times 10^{9} \mathrm{erg}$ $s^{-1} \mathrm{~cm}^{2}$, respectively. Nevertheless, the direct relation of the presence or absence of a thermal inversion layer with the incident radiation level could be confirmed since several exoplanets discussed in the literature do not seem to follow this trend.

\subsection{Are the stellar activity and the absence of a thermal inversion correlated?}

Knutson et al. (2010) investigated the correlation between the stellar activity and the presence of a thermal inversion in the atmosphere of hot Jupiters. They calculated the $\log \left(R_{\mathrm{HK}}^{\prime}\right)$ activity index from the CaII H and K spectral lines (at 3968 and $3933 \AA$, respectively) for 16 transiting planet-host stars with available secondary eclipse observations in the 3.6 and $4.5 \mu \mathrm{m}$ IRAC bandpasses (from Spitzer). They found that those exoplanets without a thermal inversion layer orbit the most active stars $\left(\log \left(R_{\mathrm{HK}}^{\prime}\right)>-4.9\right)$, and those with an inversion layer are associated with quiet stars $\left(\log \left(R_{\mathrm{HK}}^{\prime}\right)<-4.9\right)$. In order to explain the observed correlation, these authors suggested that the higher UV flux emitted by active stars would influence the photochemistry in hot Jupiter atmospheres and destroy the absorber responsible for the inversion layer.

From the list of transiting planet host stars with measured $\log \left(R_{\mathrm{HK}}^{\prime}\right)$ from Knutson et al. (2010), we identified nine exoplanets from the literature with observed secondary eclipse in the Ks band (table 1). To discuss this correlation of stellar activity with the temperature profile (with or without inversion), we investigated the relationship of $\log \left(R_{\mathrm{HK}}^{\prime}\right)$ with the estimated Ks-band brightness temperature, which is shown in figure 5 . From this sample, the exoplanets presenting a thermal inversion layer (diamonds) are placed in the left part of the plot, in the region of the less active or quiet stars $\left(\log \left(R_{\mathrm{HK}}^{\prime}\right)<-4.9\right)$. Those without an inversion layer (triangles) are placed on the right, in the region of the active stars $\left(\log \left(R_{\mathrm{HK}}^{\prime}\right)>-4.9\right)$.

The Ks-band brightness temperatures obtained in the CASE study are $T_{\mathrm{Ks}} \simeq 1647$ $\mathrm{K}$ and $T_{\mathrm{Ks}} \simeq 1885 \mathrm{~K}$ for WASP-10b and Qatar-1b, respectively. The activity indices $\left(\log \left(R_{\mathrm{HK}}^{\prime}\right)\right)$ for these objects are of -4.3 for WASP-10 (Maciejewski et al. 2011) and of -4.6 for Qatar-1 (Covino et al. 2013), placing both systems in the region of active stars and exoplanets without thermal inversion layer (fig. 5). 


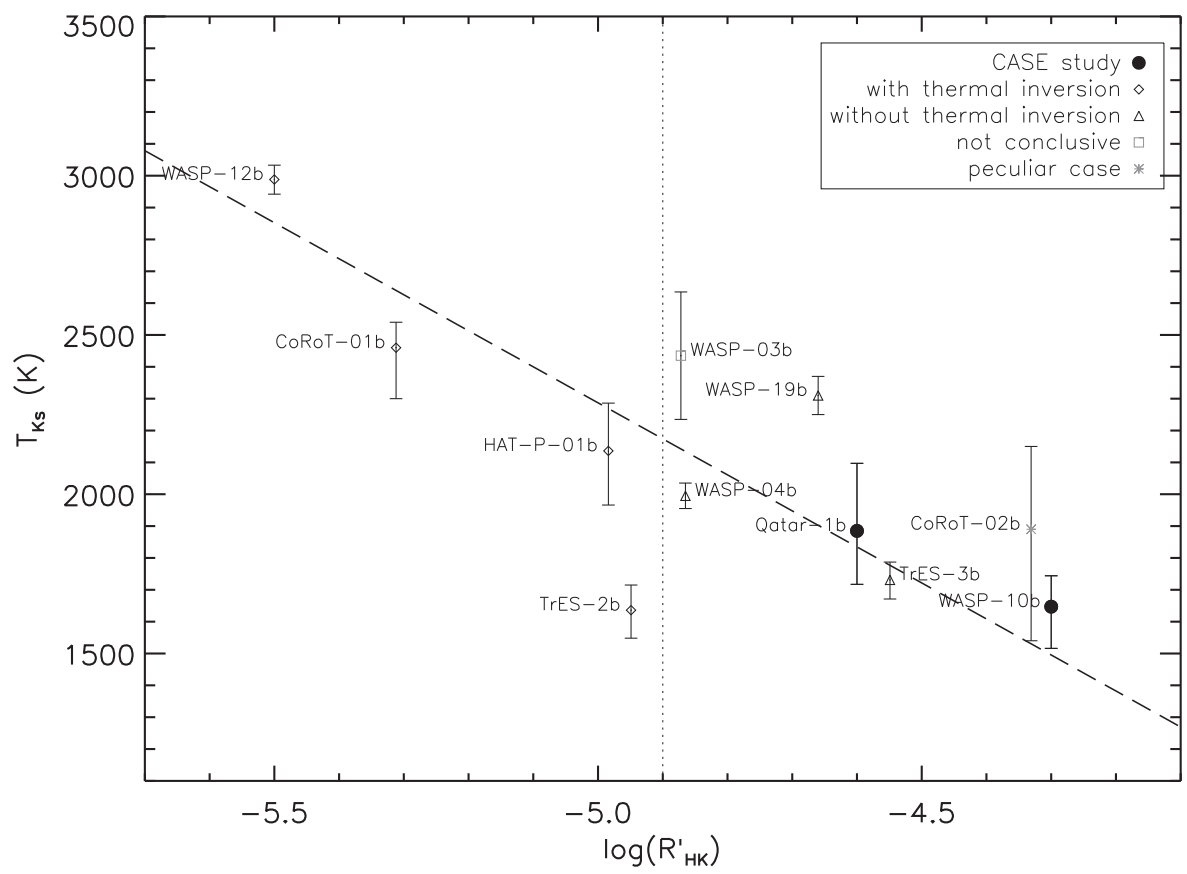

Figure 5. The $\log \left(R_{\mathrm{HK}}^{\prime}\right)$ activity index from Knutson et al. (2010) as function of the Ks-band brightness temperature from the literature. The vertical dotted line delineates the separation between active $\left(\log \left(R_{\mathrm{HK}}^{\prime}\right)>-4.9\right)$ and quiet stars $\left(\log \left(R_{\mathrm{HK}}^{\prime}\right)<-4.9\right)$. The dashed line shows an empirical correlation between both quantities with $d T_{\mathrm{Ks}} / d \log \left(R_{\mathrm{HK}}^{\prime}\right) \simeq-1131 \pm 60 \mathrm{~K}$ (Cruz 2015b).

To quantify the apparent correlation in figure 5, we performed a linear fit resulting in the following empirical relation:

$$
T_{\mathrm{Ks}}=(-3367 \pm 291)+(-1131 \pm 60) \cdot \log \left(R_{\mathrm{HK}}^{\prime}\right),
$$

with $T_{\mathrm{Ks}}$ given in $\mathrm{K}$. This empirical relationship between $\log \left(R_{\mathrm{HK}}^{\prime}\right)$ and $T_{\mathrm{Ks}}$ was based on a small number of data points (Cruz 2015b). For a more precise analysis, we need to increase the sample by measuring the activity indices of the exoplanets that have the Ks-band brightness tempertature already estimated, and to observed new secondary eclipses of known close-in hot Jupiters in the Ks band. This is an interesting correlation to be further investigated and to understand better what is driving thermal inversions in the exoplanetary atmospheres.

\section{References}

Alonso, R., Deeg, H. J., Kabath, P., \& Rabus, M. 2010, AJ, 139, 1481

Alsubai, K. A., Parley, N. R., Bramich, D. M., West, R. G., Sorensen, P. M., Collier Cameron, A., Latham, D. W., Horne, K., Anderson, D. R., Bakos, G. Á., Brown, D. J. A., Buchhave, L. A., Esquerdo, G. A., Everett, M. E., Fżrész, G., Hartman, J. D., Hellier, C., Miller, G. M., Pollacco, D., Quinn, S. N., Smith, J. C., Stefanik, R. P., \& Szentgyorgyi, A. 2011, MNRAS, 417, 709

Burrows, A., Budaj, J., \& Hubeny, I. 2008, ApJ, 678, 1436

Cáceres, C., Ivanov, V. D., Minniti, D., Burrows, A., Selman, F., Melo, C., Naef, D., Mason, E., \& Pietrzynski, G. 2011, A\& $A, 530$, A5

Christian, D. J., Gibson, N. P., Simpson, E. K., Street, R. A., Skillen, I., Pollacco, D., Collier Cameron, A., Joshi, Y. C., Keenan, F. P., Stempels, H. C., Haswell, C. A., 
Horne, K., Anderson, D. R., Bentley, S., Bouchy, F., Clarkson, W. I., Enoch, B., Hebb, L., Hébrard, G., Hellier, C., Irwin, J., Kane, S. R., Lister, T. A., Loeillet, B., Maxted, P., Mayor, M., McDonald, I., Moutou, C., Norton, A. J., Parley, N., Pont, F., Queloz, D., Ryans, R., Smalley, B., Smith, A. M. S., Todd, I., Udry, S., West, R. G., Wheatley, P. J., \& Wilson, D. M. 2009, MNRAS, 392, 1585

Covino, E., Esposito, M., Barbieri, M., Mancini, L., Nascimbeni, V., Claudi, R., Desidera, S., Gratton, R., Lanza, A. F., Sozzetti, A., Biazzo, K., Affer, L., Gandolfi, D., Munari, U., Pagano, I., Bonomo, A. S., Collier Cameron, A., Hébrard, G., Maggio, A., Messina, S., Micela, G., Molinari, E., Pepe, F., Piotto, G., Ribas, I., Santos, N. C., Southworth, J., Shkolnik, E., Triaud, A. H. M. J., Bedin, L., Benatti, S., Boccato, C., Bonavita, M., Borsa, F., Borsato, L., Brown, D., Carolo, E., Ciceri, S., Cosentino, R., Damasso, M., Faedi, F., Martínez Fiorenzano, A. F., Latham, D. W., Lovis, C., Mordasini, C., Nikolov, N., Poretti, E., Rainer, M., Rebolo López, R., Scandariato, G., Silvotti, R., Smareglia, R., Alcalá, J. M., Cunial, A., Di Fabrizio, L., Di Mauro, M. P., Giacobbe, P., Granata, V., Harutyunyan, A., Knapic, C., Lattanzi, M., Leto, G., Lodato, G., Malavolta, L., Marzari, F., Molinaro, M., Nardiello, D., Pedani, M., Prisinzano, L., \& Turrini, D. 2013, A\& A, 554, A28

Croll, B., Albert, L., Lafreniere, D., Jayawardhana, R., \& Fortney, J. J. 2010a, ApJ, 717, 1084

Croll, B., Jayawardhana, R., Fortney, J. J., Lafreniere, D., \& Albert, L. 2010b, ApJ, 718, 920

Croll, B., Lafreniere, D., Albert, L., Jayawardhana, R., Fortney, J. J., \& Murray, N. 2011, AJ, 141,30

Cruz, P., Barrado, D., Lillo-Box, J., Diaz, M., Birkby, J., López-Morales, M., Hodgkin, S., \& Fortney, J. J. 2015, A\& A, 574, A103

Cruz, P. 2015b, PhD Thesis, Universidad Autónoma de Madrid

Cruz, P., Barrado, D., Lillo-Box, J., Diaz, M., Birkby, J., López-Morales, M., \& Fortney, J. J. 2016, A\&A, 595, A61

de Mooij, E. J. W., de Kok, R. J., Nefs, S. V., \& Snellen, I. A. G. 2016, A\&A, 528, A49

Fortney, J. J., Saumon, D., Marley, M. S., Lodders, K., \& Freedman, R. S. 2006, ApJ, 642, 495

Fortney, J. J., Lodders, K., Marley, M. S., \& Freedman, R. S. 2008, ApJ, 678, 1419

Gillon, M., Demory B.-O., Barman, T., Bonfils, X., Mazeh, T., Pont, F., Udry, S., Mayor, M., \& Queloz, D. 2007, A\& A, 471, L51

Johnson, J. A., Winn, J. N., Cabrera, N. E., \& Carter, J. A. 2009, ApJL, 692, L100

Maciejewski, G., Raetz St., Nettelmann, N., Seeliger, M., Adam, C., Nowak, G., \& Neuhäuser, R. 2011, $A \& A, 535, \mathrm{~A} 7$

Mandel, K. \& Agol, E. 2002, ApJ, 580, L171

Moutou, C., Pont, F., Bouchy, F., \& Mayor, M. 2004, A\&A, 424, L31

Rogers, J., Apai, D., López-Morales, M., Sing, D., \& Burrows, A. 2009, ApJ, 707, 1707

Zhao, M., Milburn, J., Barman, T., Hinkley, S., Swain, M. R., Wright, J., \& Monnier, J. D. 2012, ApJ, 748, L8

Zhou, G., Bayliss, D. D. R., Kedziora-Chudczer, L., Salter, G., Tinney, C. G., \& Bailey, J. 2014, $M N R A S, 445,2746$ 